\title{
Mesenteric Lymphadenitis can Mimic Acute Appendicitis in Children
}

\author{
Sultan Almalki* \\ Assistant Professor, Pediatric Department, College of Medicine, Taif University, Saudi Arabia
}

*Corresponding author: Sultan Almalki, MD, Assistant Professor, Pediatric Department, College of Medicine, Taif University, Saudi Arabia, E-mail: sultanab@tu.edu.sa

Received: 25 Oct, 2021 | Accepted: 25 Nov, 2021 | Published: 01 Dec, 2021

Citation: Almalki S (2021) Mesenteric Lymphadenitis can Mimic Acute Appendicitis in Children. J Clin Case Stu 6(6): dx.doi.org/10.16966/24714925.243

Copyright: (C) 2021 Almalki S. This is an open-access article distributed under the terms of the Creative Commons Attribution License, which permits unrestricted use, distribution, and reproduction in any medium, provided the original author and source are credited.

\section{Abstract}

Abdominal pain in children is one of the most common symptoms that a child experiences in his life, and in most cases, the related causes are benign. But when pain is acute, severe and are concentrated in the lower right part of the abdomen, the surgical causes should be excluded, the most important of which is Acute Appendicitis (AA).

Usually, the diagnosis of AA is based on the findings of the clinical examination and the treatment is surgical intervention and appendectomy. However, the clinical presentation of Mesenteric Lymphadenitis (ML) can have similar symptoms of AA and does not require surgical intervention.

$\mathrm{ML}$ attributed to many causes most of which is related to viral infections or bacterial infections in less cases, but the treatment here is a medical treatment only by prescribing pain relievers or using antibiotics if it is caused by a bacterial infection. Therefore, it is necessary to differentiate between these cases and make sure of the diagnosis so that any unnecessary surgical intervention can be avoided. Ultrasound examination is one of the most important methods of diagnosis.

Herein, we describe a case of a 12-year-old child who was complaining of symptoms like those of the AA, and based on clinical evaluation, the initial decision was surgical intervention and removal of the appendix. But by conducting some additional tests, the most important of which is the ultrasound, it was found that he suffers from enlargement and inflammation in the abdominal lymph nodes, which treated successfully medically without resorting to surgical intervention. Accordingly, we conclude the importance of performing the abdominal ultrasound in such cases to ascertain and differentiate between the two diseases and thus reach a successful treatment method.

\section{Introduction}

Abdominal pain is one of the most common presenting symptoms of children brought to medical care and comprises a differential diagnosis of various etiologies [1]. Amongst children looking for medical care in emergency departments or outpatient clinics with abdominal pain, acute appendicitis (AA) is the most frequent surgical etiology, the treatment usually is invasive and require surgical intervention [2]. However, mesenteric lymphadenitis (ML) is considered as one of the most common differential diagnosis in a child with suspected acute appendicitis, the treatment here mainly is supportive care with adequate hydration and pain killer with nonsteroidal anti-inflammatory medications [3]. Therefore, it is crucial to differentiate between the two conditions to avoid needless surgery.

Mesenteric lymphadenitis is common presentation in pediatric populations. When a child who are under 10 years of age exhibits acute right lower quadrant abdominal pain is more likely to have mesenteric lymphadenitis rather than acute appendicitis. Mesenteric lymphadenitis frequently associated with gastrointestinal or upper respiratory infections [4]. The clinical presentation encompasses fever, vomiting, change in bowel habits, right lower quadrant abdominal pain and tenderness [5]. The pathology can be described as an inflammatory process of the mesenteric lymph nodes that can be of primary (idiopathic) etiology or secondary to numerous infective, malignant, or inflammatory disorders. It is commonly defined as a collection of three or more lymph nodes visualized on CT scan or US measuring more than $5 \mathrm{~mm}$ on their short axis [6]. Nowadays, the diagnosis is made commonly when computed tomography or ultrasonography is executed to assess a patient with suspected acute appendicitis [7].

In this case study, we describe a 12 -year-old boy presented with acute right lower quadrant abdominal pain mimic acute appendicitis clinical presentation. Nevertheless, by help of abdominal US, the diagnosis turned to be benign primary ML and treated successfully with supportive therapy and antibiotics without surgical intervention.

\section{Case Description}

A 12-year-old male who did not suffer from previous health problems and is leading his life normally, but he suddenly experienced severe pain in the abdomen for one day, which called him to go to the emergency room. The abdominal pain was accompanied by a moderate rise in temperature, nausea, and vomiting for several times. The pain was concentrated on the right side and the lower part of the abdomen, and the pain increased with movement and walking. There 
was a loss of appetite and he had passed stool twice on the same day, as his usual. The parents denied the presence of urinary symptoms or other systemic symptoms.

\section{On examination}

The child was fully conscious but felt severe pain in the lower abdomen on the right side when touching it, and the pain increased by moving the right thigh. The child was in a bowed position to reduce the pain. The vital signs were stable, and no signs of dehydration. The clinical assessment was suggestive for the presence of acute appendicitis. Based on that, basic blood work, urine analysis, and abdominal ultrasound were performed to rule out appendicitis.

\section{Investigation}

- Lab test has been consistent with viral infection, and revealed $\mathrm{CBC}$ with normal WBC count with lymphocyte predominant and CRP is not elevated.

- Ultrasound examination of the abdomen revealed the presence of inflammation and enlargement of several mesenteric lymph nodes, with normal appearance of the appendix. The view of the liver, spleen and kidneys were normal (Figure 1,2).

\section{Treatment}

Child treated with encouraging oral fluid intake, paracetamol, and oral broad-spectrum antibiotic.

\section{Discussion}

Mesenteric lymphadenitis (ML) is considered as one of the most common differential diagnosis in a child with possible acute appendicitis (AA). Therefore, Mesenteric lymphadenitis and acute appendicitis should be differentiated by multiple clinical and laboratory parameters [1].

In a retrospective study, the clinical and laboratory results of patients diagnosed with ML were compared to patients diagnosed with AA. It found that patients with ML had significantly longer duration of symptoms prior to emergency department (ED) presentation and had longer duration of stay in the ED [1]. However, in our case the duration was acute for one day and had no admission. On the same study, patients with ML also had significantly lower WBC with lymphocyte predominance and lower CRP levels. Moreover, the study showed that vomiting, and classic abdominal findings of AA were all significantly more common for children with AA. In contrast, the vomiting and abdominal pain in our case was also suggestive for AA initially. However, after performing abdominal US the findings become suggestive more of primary ML rather than AA, supported by presence of abdominal pain with mesenteric LN enlargement and intact appendix. Primary mesenteric lymphadenitis is a self-limiting inflammatory disorder affecting three or more of mesenteric lymph nodes with no secondary pathology [8]. Likewise, the findings of US in our case showed enlargement of LN without obvious secondary cause.

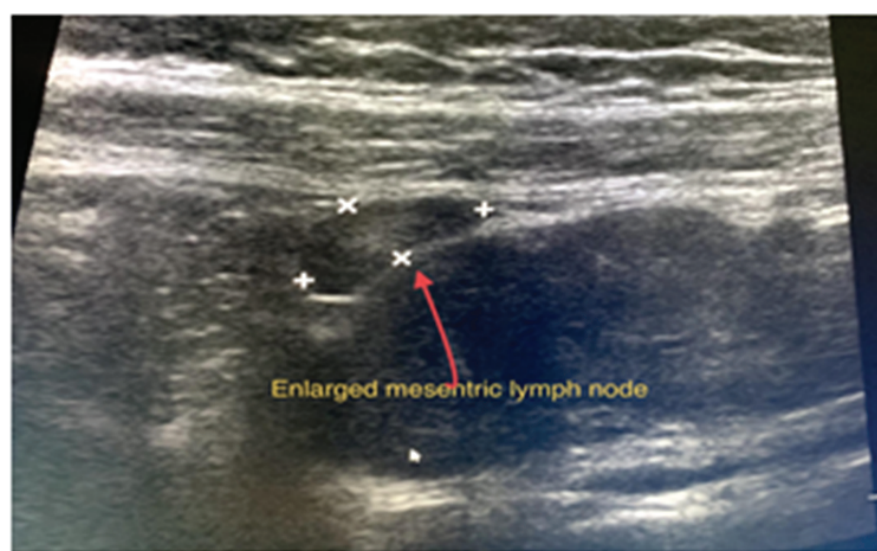

A

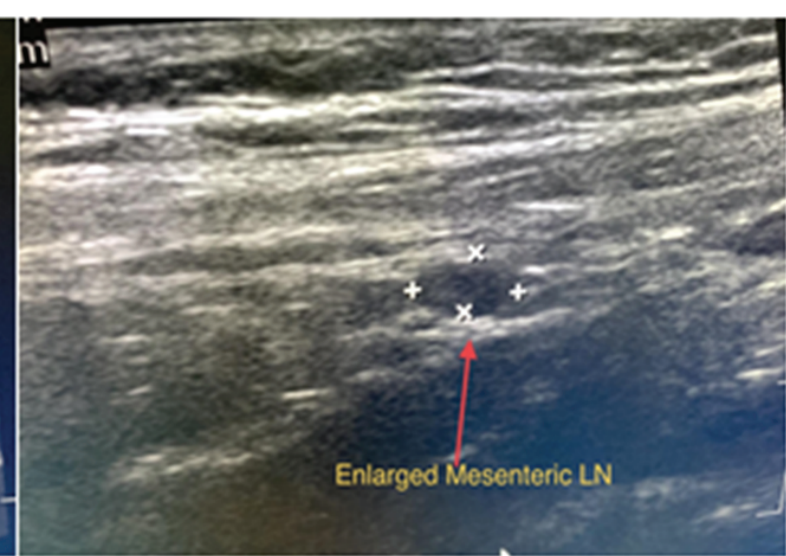

B

Figure 1: Abdominal US (A) and (B) showing Mesenteric Lymphadenopathy.

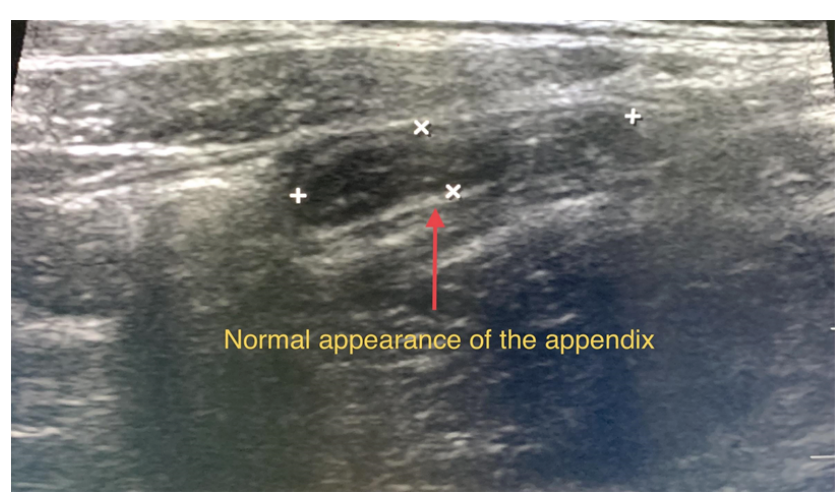

Figure 2: Abdominal US showing normal appearance of Appendix.

Citation: Almalki S (2021) Mesenteric Lymphadenitis can Mimic Acute Appendicitis in Children. J Clin Case Stu 6(6): dx.doi. org/10.16966/2471-4925.243 
In another study, it found that abdominal pain was the most prevalent complaint in children with mesenteric lymphadenopathy and showed that most of the patients had long axis of the lymph nodes reached minimum $10 \mathrm{~mm}$ [9]. It also found that primary mesenteric lymphadenopathy was the most frequent diagnosis [9].

Identification of right lower quadrant mesenteric nodes in patients with acute abdominal pain is usually associated with intraperitoneal inflammatory condition in most patients. Inability to recognize a concurrent pathologic process leads to a possible diagnosis of primary mesenteric lymphadenitis and explains the patient's symptoms [4]. In secondary mesenteric lymphadenitis, reactive mesenteric lymph node enlargement can arise from localized or systemic inflammatory processes. Acute mesenteric lymphadenitis also can occur because of bacterial infection such as Yersinia pseudotuberculosis and Yersinia enterocolitica, Bartonella henselae, Salmonella spp [1].

Ultrasound imaging is invaluable in detecting ML and finding the alternative diagnosis. A study by Lim JK, et al. advocated the usage of ultrasonography in the diagnosis of mesenteric lymphadenitis [10]. Ultrasound had a positive predictive value of $96 \%$ for acute appendicitis in another study [5]. Ultrasonography can diagnose the primary LM if it reveals 3 or more mesenteric lymph nodes without any recognizable underlying inflammatory cause [11]. In a published study by Kaur I, et al. demonstrated the upper limits of normal size varying by up to $5 \mathrm{~mm}$ in adults and $8 \mathrm{~mm}$ in children. However, the imaging features of enlarged nodes cannot differentiate lymphadenitis due to primary infection or secondary to other inflammatory processes [7]. In another study by Wang WG, et al. described that the lymph nodes with a short diameter larger than $8 \mathrm{~mm}$ is suggestive for mesenteric lymphadenitis [11]. Similarly, Karmazyn B, et al. have demonstrated that short-axis diameter of $8 \mathrm{~mm}$ as the upper limit of normal mesenteric lymph node size in children [12]. Moreover, Sheridan AD, et al. showed the potential value of ultrasonography to stratify patients with acute appendix into different treatment strategies [13].

\section{Conclusion}

It is difficult to distinguish acute mesenteric lymphadenitis from acute appendicitis in children based on clinical evaluation alone. Ultrasound should be performed to confirm the diagnosis and specify the treatment. Thus, we can avoid unnecessary surgical intervention in children with mesenteric lymphadenitis cases.

\section{References}

1. Gross I, Siedner-Weintraub Y, Stibbe S, Rekhtman D, Weiss D, et al. (2017) Characteristics of mesenteric lymphadenitis in comparison with those of acute appendicitis in children. Eur J Pediatr 176: 199-205.
2. Kulik DM, Uleryk EM, Maguire JL (2013) Does this child have appendicitis? A systematic review of clinical prediction rules for children with acute abdominal pain. J Clin Epidemiol 66: 95-104.

3. Puylaert JB, van der Zant FM (1995) Mesenteric lymphadenitis or appendicitis? AJR Am J Roentgenol 165: 490.

4. Otto M, Nagalli S (2021) Mesenteric Adenitis. In: StatPearls. Treasure Island (FL): StatPearls Publishing.

5. Toorenvliet B, Vellekoop A, Bakker R, Wiersma F, Mertens B, et al.(2011) Clinical differentiation between acute appendicitis and acute mesenteric lymphadenitis in children. Eur J Pediatr Surg 21: 120-123.

6. Macari M, Hines J, Balthazar E, Megibow A (2002) Mesenteric adenitis: CT diagnosis of primary versus secondary causes, incidence, and clinical significance in pediatric and adult patients. AJR Am J Roentgenol 178:853-858.

7. Kaur I, Long SS (2019) 50 Years Ago in The Journal of Pediatrics: Acute Mesenteric Lymphadenitis. J Pediatr 206: 211.

8. Helbling R, Conficconi E, Wyttenbach M, Benetti C, Simonetti GD, et al. (2017) Acute Nonspecific Mesenteric Lymphadenitis: More Than “No Need for Surgery." Biomed Res Int 2017: 9784565.

9. Sikorska-Wiśniewska G, Liberek A, Góra-Gebka M, Bako W, Marek A, et al. (2006) Mesenteric lymphadenopathy - a valid health problem in children. Med Wieku Rozwoj 10: 453-462.

10. Lim KJ, Lee K, Yoon DY, Moon JH, Lee H, et al. (2015) The role of US in finding intussusception and alternative diagnosis: a report of 100 pediatric cases. Acta Radiol 56: 228-233.

11. Wang WG, Tian H, Yan JY, Li T, Zhang TD, et al. (2011) Enlarged mesenteric lymph nodes in children: a clinical analysis with ultrasonography and the implications. Nan Fang Yi Ke Da Xue Xue Bao 31: 522-524.

12. Karmazyn B, Werner EA, Rejaie B, Applegate KE (2005) Mesenteric lymph nodes in children: what is normal? Pediatr Radiol 35: 774777.

13. Sheridan AD, Ehrlich L, Morotti RA, Goodman TR (2015) Sonographic distinction between acute suppurative appendicitis and viral appendiceal lymphoid hyperplasia ("pink appendix") with pathological correlation. Ultrasound Q 31: 95-98. 